\title{
MOOC: Internet y Personas Mayores
}

\section{Carlos Hernández Franco ${ }^{\mathrm{a}}$ y Carmen García Ferrando ${ }^{\mathrm{a}}$}

${ }^{a}$ Escuela Técnica Superior de Ingenieros de Telecomunicación, Universitat Politècnica de València, chernan@dcom.upv.es, cargarf3@teleco.upv.es

\begin{abstract}
The appearance over two decades of the Information and Communications Technology and the development and widespread use of Internet has brought a revolution in the forms, modes, contents, scope, immediacy, etc. to perform virtually all processes and relationships in the contemporary society. The effective use of tablets and smartphones provide a useful way of "socializing" that would result in a substantial improvement in the quality of life and welfare of the elder people. The Universidad Senior at Universitat Politècnica de València offers from the academic course 2009-2010 the monographic course "Useful Internet" where elder people is oriented on the use of Internet and its many services anytime, anywhere (mobility culture). The creation of "MOOC: Internet and the elder people" in UPV[X] (the repository for Massive Online Open Courses or MOOC) has among its main objectives to support the monographic course "Useful Internet", while contributing to the widespread use of mobile Internet among elder people anywhere and anytime. This is a fundamental aspect of the social responsibility of our university.
\end{abstract}

Keywords: Internet, Elder People, Universidad SENIOR UPV, MOOC

\footnotetext{
Resumen

La aparición hace ya más de dos décadas de las llamadas Tecnologías de la Información y la Comunicación así como el desarrollo y uso generalizado de Internet, ha supuesto una revolución en las formas, los modos, los contenidos, el alcance, la inmediatez, etc., de realizar prácticamente todos los procesos e interrelaciones de la sociedad contemporánea. Disponer y utilizar de formar eficaz tablets y smartphones constituirá un magnifico medio "socializador" que redundaría en una mejora sustancial de la calidad de vida y el bienestar de las personas mayores.
} 
MOOC: Internet y Personas Mayores

La Universidad Senior UPV ofrece desde el Curso 2009-2010 el monográfico "Internet Útil" donde se orienta a personas mayores en el acceso a Internet y sus múltiples servicios en cualquier momento y desde cualquier lugar (cultura de la movilidad). La creación del "MOOC: Internet y Personas Mayores” en UPV[X] (el repositorio de Cursos Masivos Online de la UPV) tiene entre sus objetivos principales el servir de apoyo al monográfico "Internet Útil", a la vez que contribuye a la difusión del uso de Internet móvil entre las personas mayores en cualquier lugar y en cualquier momento. Esto último, constituye un aspecto fundamental de la responsabilidad social de nuestra Universidad.

Palabras clave: Internet, Personas Mayores, Universidad Senior UPV, MOOC

\section{Introducción}

La aparición hace ya más de dos décadas de las llamadas Tecnologías de la Información y la Comunicación así como el desarrollo y uso generalizado de Internet, ha supuesto una revolución en las formas, los modos, los contenidos, el alcance, la inmediatez, etc., de realizar prácticamente todos los procesos e interrelaciones de la sociedad contemporánea. Asimismo, la "conectividad” entre regiones, países, hemisferios y culturas se ha visto posibilitada e impulsada como nunca antes lo había logrado el género humano.

Claro está, muchas ventajas y también desventajas han ido apareciendo durante todo este tiempo. Siendo incluso necesario el acuñar nuevos vocablos o expresiones para definir mejor este nuevo contexto tecnológico social: sociedad "hiperconectada", “postdigitalismo”, “brecha digital”, “nativos digitales”, etc. (Castells 2001).

Estudios consultados demuestran que todo el desarrollo asociado con este contexto afecta en mayor medida a las personas mayores, debido fundamentalmente a su inexperiencia tecnológica (AEPUM 2015).

Con la aparición de tablets y smartphones surgen nuevas problemáticas relacionadas con el costo de los terminales y sus dimensiones, la obsolescencia programada, o el concepto de las aplicaciones que deben instalar para disfrutar de determinados servicios siendo a veces un verdadero problema. Sin embargo, disponer y utilizar de formar eficaz dispositivos de este tipo, constituiría un magnifico medio “socializador” que redundaría en una mejora sustancial de la calidad de vida y el bienestar de las personas mayores (Minetur 20015).

(c)) EY-NC-ND 2015, Universitat Politècnica de València 
La Universidad Senior UPV ofrece desde el Curso 2009-2010 el monográfico "Internet Útil" donde se orienta a personas mayores en el acceso a Internet, y sus múltiples servicios, en cualquier momento y desde cualquier lugar (cultura de la movilidad) haciendo uso para ello de tablets y smartphones. La creación del "MOOC: Internet y Personas Mayores" en UPV[X] (el repositorio de Cursos Masivos Online de la UPV) tiene entre sus objetivos principales el servir de apoyo al monográfico "Internet Útil", a la vez que contribuye a la difusión del uso de Internet móvil entre las personas mayores en cualquier lugar y en cualquier momento. Esto último, un aspecto fundamental de la responsabilidad social de nuestra Universidad (UPV 2015).

\section{Objetivos}

La Universidad Senior UPV ofrece el monográfico “Internet Útil” donde el objetivo principal es que las personas mayores reciban la formación acerca de Internet y sus múltiples servicios.

Uno de los objetivos más importantes de dicho monográfico es la concienciación de la cultura de la movilidad, ya que hoy en día estamos conectados permanentemente desde casi cualquier dispositivo: smartphones, tablets, portátiles, etc. Se pretende mostrar todo el mundo de posibilidades que nos ofrece la red, y favorecer de esta manera la inclusión de las personas mayores en la sociedad actual.

Este monográfico consta de 10 sesiones y cada una de ellas de 2 horas, por lo que es beneficiosa la creación de un MOOC "Internet y Personas Mayores” como refuerzo a dicho curso. De esta forma los alumnos pueden obtener una visión mucho más amplia y más tranquila sobre los contenidos que han recibido de forma presencial.

Las principales funciones que se desean cubrir con el MOOC "Internet y Personas Mayores" son las de poder "repasar" de forma autónoma todo lo visto de una manera tranquila y relajada. Los alumnos pueden tomar el MOOC a modo de "apuntes", los que por supuesto pueden consultar en cualquier momento y lugar.

Asimismo, resulta interesante la idea de MOOC, ya que su filosofía (su significado es "Cursos masivos abiertos on line”) es precisamente la de la máxima difusión posible, de una manera gratuita, mediante una plataforma educativa, como es en este caso la UPV.

Se consiguen cumplir dos objetivos a la vez, el apoyo a los alumnos del curso de la Universidad Senior de la UPV y además, que esa información pueda ser también consultada por cualquier persona interesada en el tema, consiguiendo así una liberación de conocimientos.

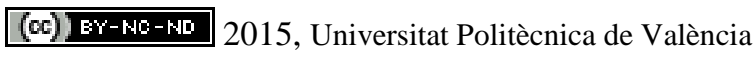

Congreso In-Red (2015): 

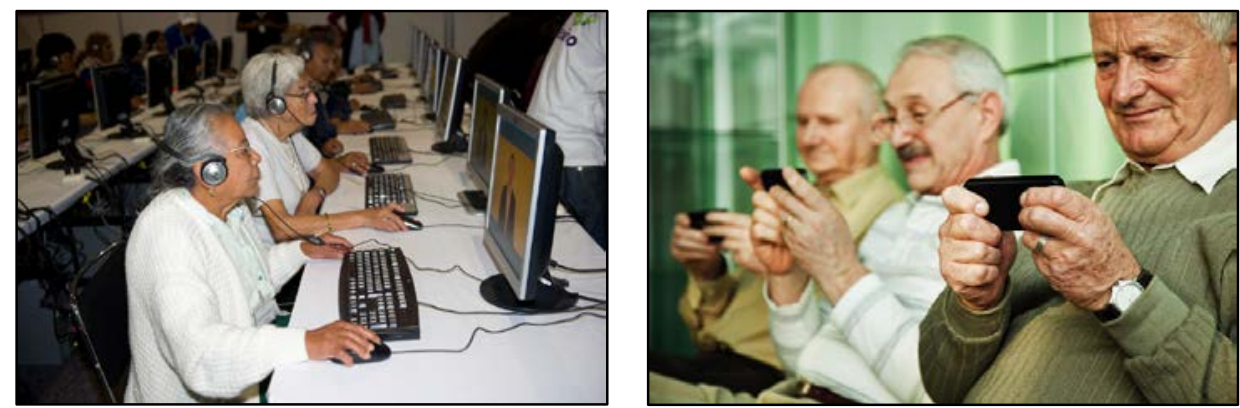

Fig. 1 Tecnologías de la Información y las Personas Mayores.

\section{Metodología}

Para poder adecuar el contenido del MOOC "Internet y Personas Mayores" se han tomado como base los temas del monográfico "Internet Útil", el cual, como se ha dicho anteriormente, lleva impartiéndose desde el curso 2009-2010. Hemos adquirido cierta experiencia durante todos estos cursos, lo que nos ha permitido elegir y tratar mejor los contenidos incluidos en el MOOC. Aún con esto, decidimos perfilar mejor el alumno al cual va dirigido y para ello realizamos una encuesta on line.
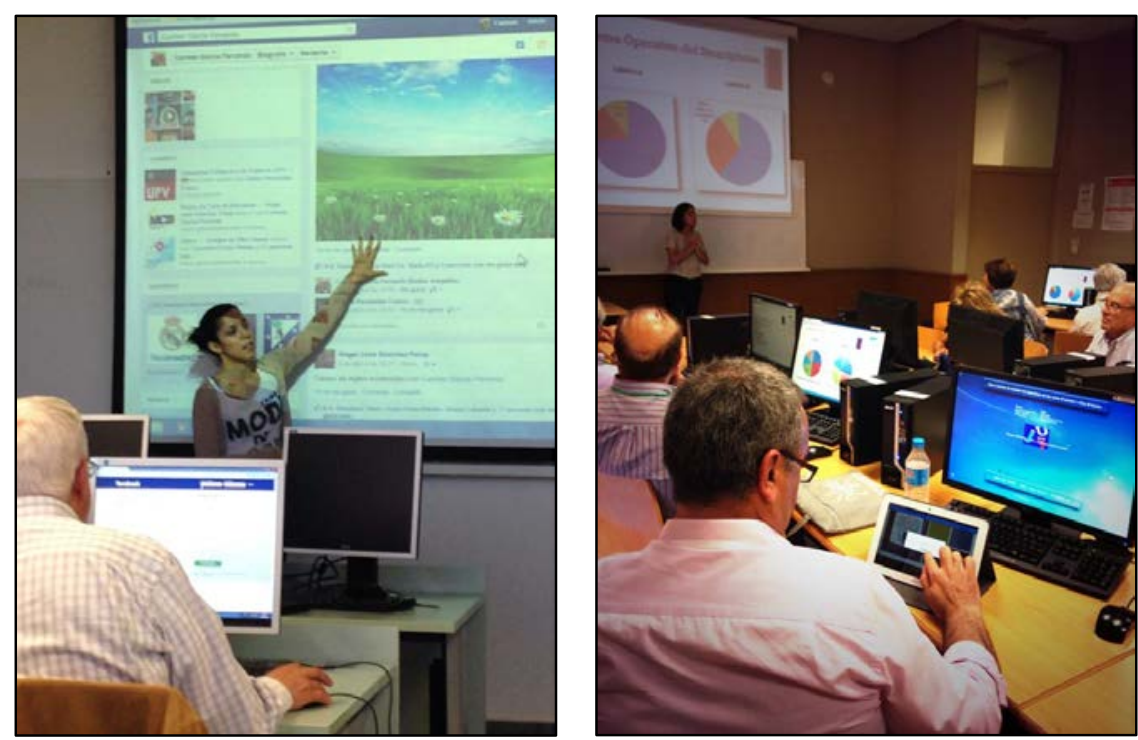

Fig. 2 Monográfico sobre Tecnologías de la Información para las Personas Mayores.

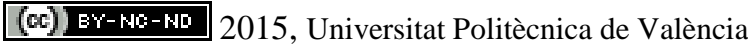
Congreso IN-RED (2015): 
Un aspecto a tener en cuenta cuando de estos temas se trata, en este caso Internet, es el de su constante evolución, los cambios se suceden de un día para otro y de manera muy drástica. Por este motivo, el monográfico ha ido evolucionando desde su comienzo al ritmo de la evolución de la red. Por ello cabe señalar que el MOOC, en este sentido, resulta de fácil adaptación ya que simplemente bastaría incluir los nuevos cambios con nuevo material. Actualmente, dicho MOOC se encuentra en proceso de realización, estando prevista su puesta en marcha de cara al próximo Curso Académico 2015-2016.

\subsection{Encuesta on line a los alumnos de la Universidad Senior UPV}

Con el objetivo de poder personalizar los contenidos del MOOC de acuerdo con el público al cual va dirigido, se decidió realizar una encuesta, no solamente a los alumnos del monográfico "Internet Útil”" sino a todos los miembros de la Universidad Senior de la UPV, independientemente del curso en el que estuvieran matriculados, ya que uno de los objetivos a cumplir con el MOOC, es el de la liberación de conocimientos, que cualquier persona que esté interesada en el tema pueda acceder a los contenidos. Por este motivo se toma como perfil las personas mayores que acuden a nuestra Universidad.

Para realizar la encuesta se hizo uso de un portal on line, www.portaldeencuestas.com, y a través del Vicerrectorado de Responsabilidad Social y Cooperación de la UPV, se envió a 1800 alumnos de la Universidad Senior obteniéndose 521 respuestas. Una vez finalizado el período de realización, se procedió al análisis de los datos obtenidos algunos de los cuáles detallamos a continuación.

Resulta fundamental destacar el marco en el que la misma fue realizada, es decir, hay que considerar que las personas encuestadas viven en su gran mayoría en la ciudad de Valencia y que tienen cierto nivel de estudios y "sentimiento" cultural, lo que les hace establecer relación más próxima con la Universidad, con tal de seguir formándose y mantenerse activos. Esta misma encuesta, realizada en un marco totalmente diferente, por ejemplo en una población significativamente más pequeña, daría resultados, seguramente, bien distintos a los obtenidos.

Dicho esto, si pasamos ya a analizar algunos resultados obtenidos, encontramos en primer lugar cuál es el dispositivo de conexión más comúnmente empleado entre las Personas Mayores. Como era de esperar, debido a la cultura de la movilidad, el dispositivo mediante el cual se conectan es el terminal móvil o "smartphone", seguramente porque lo llevan consigo a casi cualquier parte y es lo que resulta más cómodo. Siguen siendo bastante comunes el ordenador de sobremesa y el ordenador portátil. En la figura 3 podemos observar que elementos como el "phablet" están prácticamente en desuso en este sector, quizá por el desconocimiento de su significado.

\section{(c) EY-NC-ND 2015, Universitat Politècnica de València}




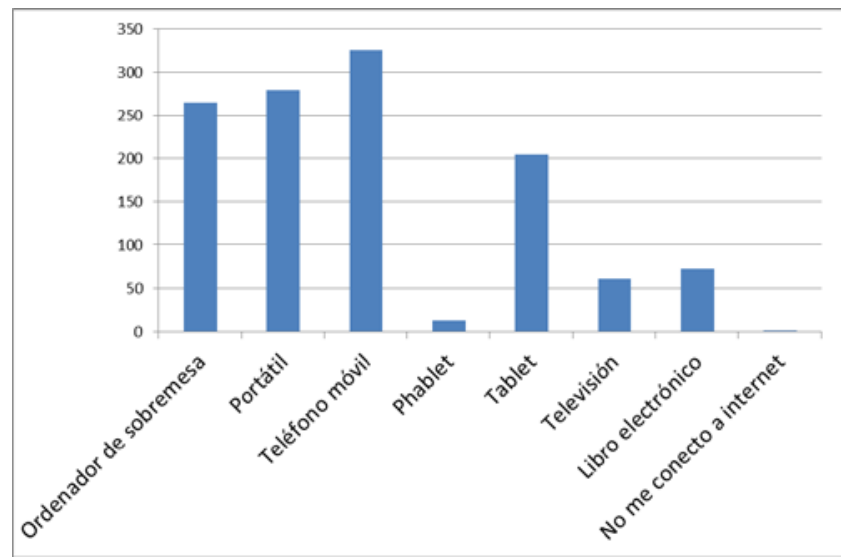

Fig. 3 Dispositivos de conexión a Internet.

Otro aspecto importante, una vez conocido el dispositivo de conexión, es el motivo de ésta. ¿Qué lleva a este tipo de perfil a conectarse a Internet? En la figura 4 podemos ver que los principales motivos son la búsqueda de información y la consulta del correo electrónico. Lo que nos lleva a pensar que es un sector de la población consciente de las ventajas que puede encontrar en la red, y se dispone a buscar información de cualquier tipo. Suelen ser menos comunes las compras y el uso de las redes sociales, debidos ambos casos principalmente a la desconfianza que estos nuevos "canales".

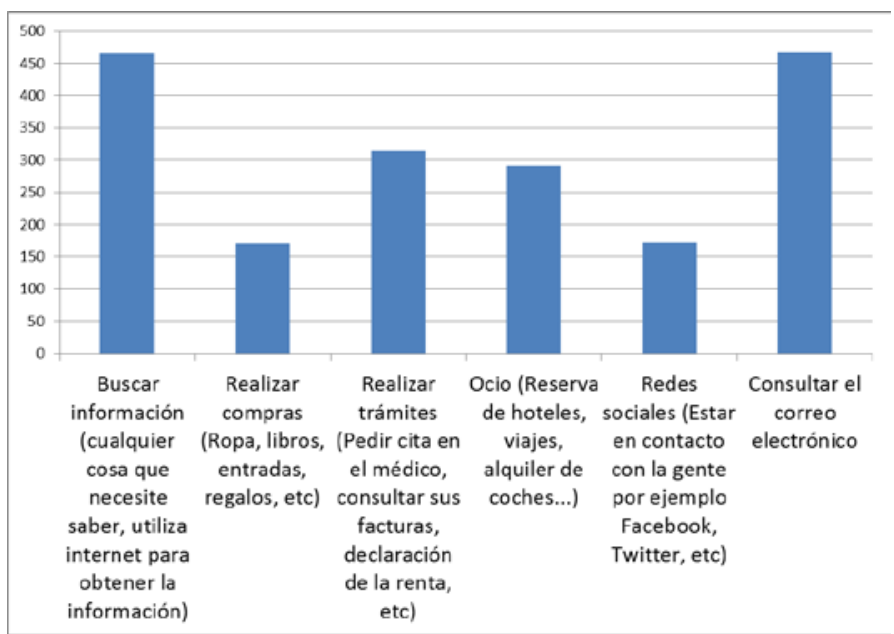

Fig. 4 Finalidad de la conexión.

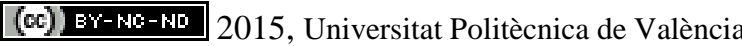


Para poder saber el grado o nivel de conocimiento de los alumnos sobre el dispositivo móvil que utilizan (smartphone y/o tablet), se preguntó sobre cuál sistema operativo utilizaba. En la figura 5 se observa que existe un porcentaje significativo de ellos que no saben qué sistema operativo posee, pero por lo demás las respuestas obtenidas son acordes a los resultados globales, que señalan al sistema Android como el más común entre los usuarios.

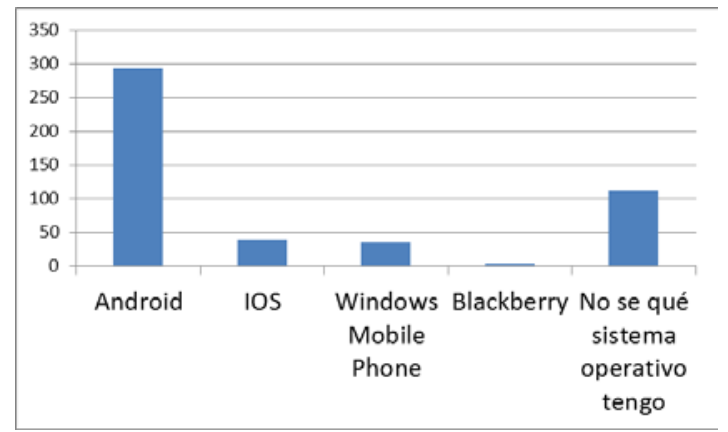

Fig. 5 Sistemas operativos del "smartphone".

Por último, y para poder desarrollar en detalle los contenidos del MOOC, se preguntó acerca de los servicios y aplicaciones (Apps) más utilizados, pudiendo incidir así durante el curso en los menos conocidos. De acuerdo con los resultados, y que coincidieron en gran medida con lo esperado por nuestra experiencia anterior, los servicios y Apps más conocidos resultaron ser Whatsapp, Google Maps y Wikipedia y los menos conocidos Doodle y aplicaciones sobre deporte tales como Runtastic y Endomondo, figura 6.

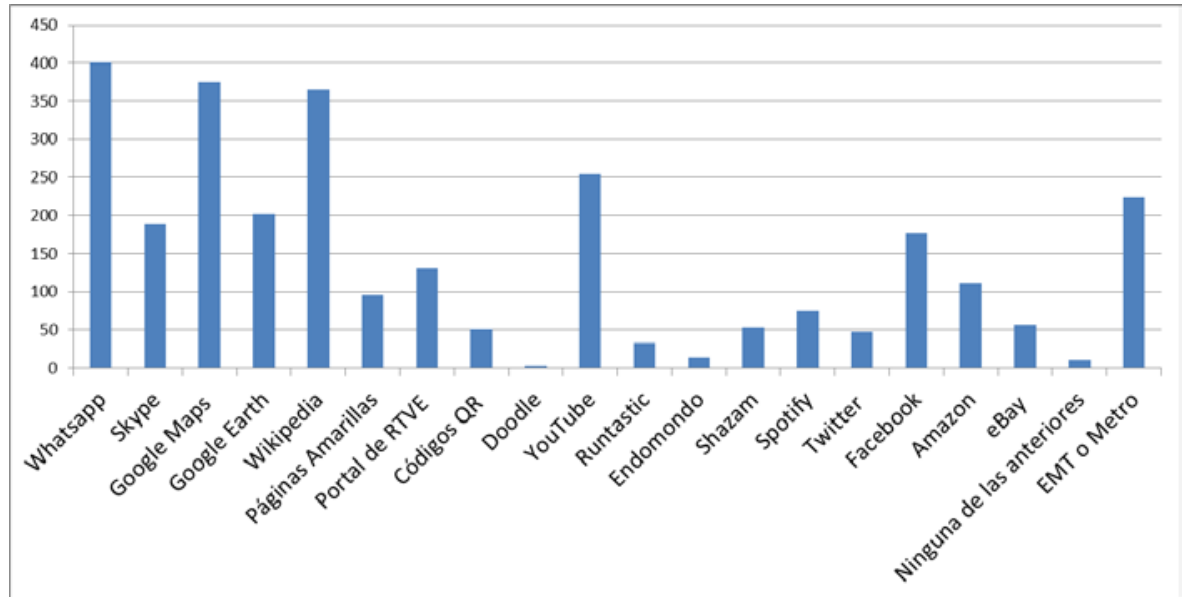

Fig. 6 Uso de servicios o "Apps".

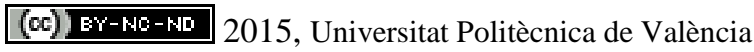

Congreso In-Red (2015): 
MOOC: Internet y Personas Mayores

\subsection{Unidades didácticas del “MOOC: Internet y Personas Mayores”}

Basándonos en los análisis de los resultados obtenidos de la encuesta online así como en nuestra propia experiencia de varios cursos académicos impartiendo el monográfico "Internet Útil”, es que se proponen y desarrollan las unidades didácticas que conforman el MOOC “Internet y Personas Mayores”.

\section{0.- Justificación encuesta y contenidos}

Es una unidad cero, un punto de partida, una justificación de la realización de dicho MOOC. Asimismo, se hace una reflexión de la evolución de nuestra forma de comunicarnos actual y cómo ha ido cambiando en el tiempo, sobre todo en estos últimos 20 años.

\section{1.- La Red de Redes}

En este tema se aborda el concepto de "Internet" que muchas veces se da por supuesto y que su concepto difuso dificulta la comprensión de elementos y servicios posteriores.

\section{2.- Conectándonos}

Se exponen diversos dispositivos de conexión y aparecen conceptos no muy conocidos como el de "phablet". También se explica cómo elegir, si bien no existe una "receta" única un smartphone o un tablet.

\section{3.- La IoT}

Esta unidad ofrece una visión de hacia dónde se encamina la tecnología y sus diversas aplicaciones. En el futuro próximo Internet estará presente en nuestro día a día mucho más, incluso en nuestro hogar, pudiendo "controlar" casi cualquier cosa a distancia a través de la red. Es lo que se denomina la "Internet de las Cosas" o IoT.

\section{4.- La nube}

Este es otro concepto novedoso y muy difuso para las Personas Mayores y que cada día más se viene escuchando a través de los medios de comunicación.

\section{5.- Las contraseñas}

Explicar cómo poder organizar nuestras contraseñas, reglas para poder establecerlas y recordarlas. Consejos sobre que es mejor si tener la misma contraseña para todos nuestros accesos en la red o diferentes para cada uno de ellos. Se evalúan los pros y los contras de todo lo anterior. 


\section{6.- La privacidad}

Cuáles son las huellas que dejamos en Internet y cómo podemos protegernos frente a ataques hacia nuestra privacidad.

\section{7.- Programas vs Aplicaciones}

Se intenta explicar la principal diferencia entre lo que es un programa y lo que es el nuevo concepto de aplicación.
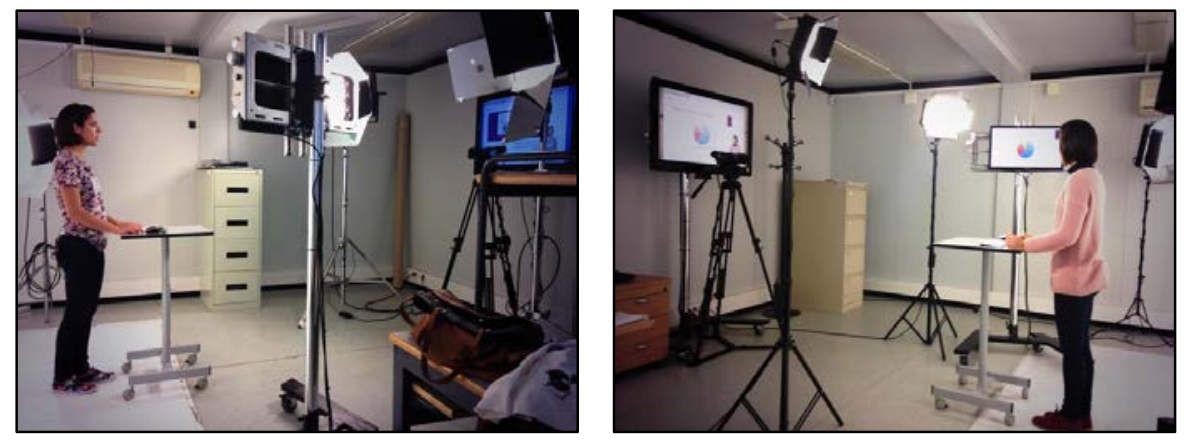

Fig. 7 Grabación de contenidos para el MOOC utilizando la tecnología Polimedia.

\section{8.- Información}

Se establece una selección de servicios y Apps agrupados en cuatro grandes bloques. El primero de ellos es el bloque de Información donde se engloban aplicaciones como lectores de códigos QR, el estado del tiempo, el acceso a contenidos de Radio y TV, medios públicos de transporte, etc.

\section{9.- Ocio}

Una selección sobre ocio, sobre todo para este sector que normalmente son personas ya jubiladas y que demandan este tipo de servicios. Se hace referencia a Doodle, venta de entradas, guía de restaurantes, categorización de vinos, fotografía digital, deporte, música y por supuesto el uso de las redes sociales.

\section{0.- Compras}

En esta unidad se tratan servicios de viajes, vuelos, billetes de tren, páginas de compras con descuentos y "ofertas de último minuto", páginas para comprar a través de Internet. Se pretende explicar que las compras por Internet se pueden hacer de forma segura, por ejemplo a través del servicio de pago PayPal. 


\section{1.- Trámites}

Otro de los temas que más preocupa a las Personas Mayores es la realización de trámites a través de la red, por ello se analiza el portal de la Generalitat Valenciana, el portal del Ayuntamiento de Valencia y el portal del ciudadano (antiguo 060). Esta unidad tiene como principal objetivo dar a conocer procedimientos electrónicos que agilizan los trámites, ahorran tiempo y evitan desplazamientos innecesarios.

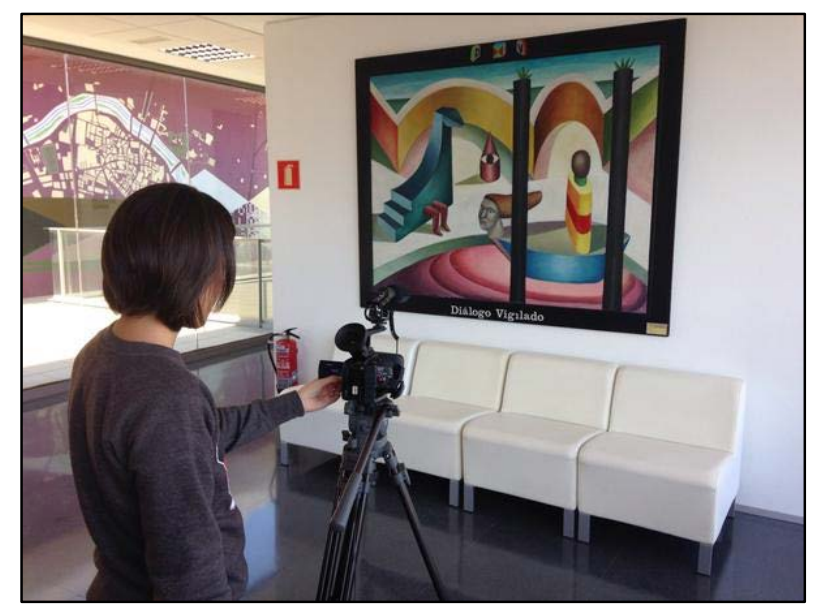

Fig. 8 Grabación de contenidos para el MOOC en "exteriores".

\section{Resultados}

El MOOC “Internet y Personas Mayores” se encuentra actualmente en fase de realización. Se espera que todo el contenido elaborado pueda servir de apoyo a los alumnos del monográfico "Internet Útil” de manera que puedan asimilar conocimientos que no resultan sencillos para ellos, de una forma mucho más cómoda, ya que pueden visualizar el contenido del monográfico desde casa.

Además, puede resultar muy útil para responder a todas aquellas nuevas dudas y preguntas que pueden ser planteadas en el aula y que por motivos de tiempo no puedan ser respondidas o analizadas en profundidad. Se espera asimismo que sirva como método de enseñanza para todas aquellas personas que estén interesadas en formarse en este campo. Y de motivación para todos aquellos alumnos que por diversos motivos no pueden asistir al monográfico ofrecido por la Universidad Senior de la UPV. 
Finalmente, esperamos cumplir los objetivos de la "filosofía" del MOOC, lograr esa "liberación de conocimientos" y poder llegar al máximo público posible, de manera que cualquiera tenga acceso a la formación sobre nuevas tecnologías, favoreciendo así la inclusión social de las Personas Mayores.

\section{Conclusiones}

Esta propuesta pone de manifiesto el creciente interés por parte de las Personas Mayores en el uso de Internet y sus innumerables y crecientes servicios. Lo que en un principio podía ser tan sólo una suposición, vemos a través de la encuesta y de los monográficos realizados que es una situación real. Este es sólo el principio de lo que puede suponer el final de la "brecha digital". La difusión de programas dedicados a la formación tecnológica de Personas Mayores puede disminuir esta “distancia” o "separación” existente entre diferentes generaciones, ayudando así a una independencia mayor por parte de este perfil de usuarios. Estas conclusiones nos pueden servir como impulso para ser conscientes de unas necesidades existentes y además poder crear nuevas formas de enseñanza, como por ejemplo, la realización de cursos on line mediante plataformas de teleenseñanza.

\section{Referencias}

ASOCIACIÓN ESTATAL DE PROGRAMAS UNIVERSITARIOS PARA PERSONAS MAYORES (AEPUM). Estudio nacional del uso de las TICs en los programas universitarios para Personas Mayores. < http://www.aepumayores.org/es/contenido/estudio-nacional-del-usode-las-tics-en-los-programas-universitarios-para-personas-mayores> [Consulta: 29 de Mayo de 2015]

CASTELLS, M. (2001). La galaxia Internet : Reflexiones sobre Internet, empresa y sociedad. Barcelona: Plaza \& Janés.

MINISTERIO DE INDUSTRIA, ENERGIA Y TURISMO. Telecomunicaciones y sociedad de la información. <http://www.minetur.gob.es/telecomunicaciones/es-ES/Paginas/index.aspx> [Consulta: 29 de Mayo de 2015]

UNIVERSIDAD POLITÉCNICA DE VALENCIA. Plan estratégico UPV 2015-2020.

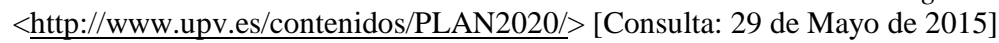

